\title{
Periodic modulation of gas giant magnetospheres by the neutral upper atmosphere
}

\author{
C. G. A. Smith \\ Atmospheric Physics Laboratory, Department of Physics and Astronomy, University College, London, UK
}

Received: 24 February 2006 - Accepted: 29 August 2006 - Published: 20 October 2006

\begin{abstract}
Periodic signatures present in the magnetospheres of both Jupiter and Saturn have yet to be fully explained. At Jupiter the unexplained signatures are related to emissions from the Io torus ("System IV"); at Saturn they are observed in emissions of kilometric radiation (SKR) and in magnetometer data. These signatures are often interpreted in terms of magnetic field anomalies. This paper describes an alternative mechanism by which the neutral atmosphere may impose such periodic signatures on the magnetosphere. The mechanism invokes a persistent zonal asymmetry in the neutral wind field that rotates with the planet. This asymmetry must be coupled to substantial ionospheric conductivity. It is then able to drive divergent currents in the upper atmosphere that close in and perturb the magnetosphere. We estimate the conductivities and wind speeds required for these perturbations to be significant, and argue that they are most likely to be important at auroral latitudes where the conductivity may be enhanced by particle precipitation.
\end{abstract}

Keywords. Ionosphere (Ionosphere-magnetosphere interactions; Planetary ionospheres) - Magnetospheric physics (Planetary magnetospheres)

\section{Introduction}

The magnetospheres of Jupiter and Saturn are dominated by the influence of the planets' rapid $\sim 10 \mathrm{~h}$ rotation periods. The principal process by which the magnetospheres are driven into rotation was described by Hill (1979) in the Jovian context (see Fig. 1). If the plasma and neutrals in the upper atmosphere rotate at different velocities, this constitutes an effective electric field that drives meridional Pedersen currents. These currents close in the magnetosphere, exerting a torque on the plasma that causes the plasma to

Correspondence to: C. G. A. Smith

(cgasmith@gmail.com) corotate, at least partially, with the planet. This is understood as the principal mechanism by which the planetary rotation period is communicated to the magnetosphere. While this process explains the partial corotation of the Jovian and Kronian magnetospheres, it is inevitable that plasma at different radial distances from the planet will acquire differing proportions of the planetary rotation period, and this is borne out by the data (McNutt et al., 1979; Richardson, 1986).

This presents difficulties in the interpretation of welldefined magnetospheric periodicities that are detected at both planets. In the case of Jupiter, numerous magnetospheric parameters exhibit a pronounced signature at the System III period $\left(\sim 9^{\mathrm{h}} 55^{\mathrm{m}}\right)$, presumably related to the considerable dipole tilt. However, a second periodicity, 3\% longer than System III, is observed in UV emissions from the Io torus. Sandel and Dessler (1988) termed this longer period System IV $\left(\sim 10^{\mathrm{h}} 13^{\mathrm{m}}\right)$. This periodicity exhibits several unexplained features. For example, Brown (1995) found that the System IV period is present only in the ion density, whereas the System III period is present in both the ion density and the ion temperature. This suggests that System IV is imposed by a different mechanism to System III.

Brown also found that the System IV period is independent of radial distance - ruling out partial corotation, which varies with radial distance, as the origin of the period - and undergoes occasional sudden phase shifts by $\sim 100^{\circ}$ in longitude. The latter effect has also been detected independently by Woodward et al. (1997), who suggested that it might be explained by System IV "features" at different longitudes whose relative magnitude varies with time. Recently, Steffl et al. (2006) have reported a periodicity in the ion composition that is only $1.5 \%$ longer than System III, complicating the situation further. It is not clear whether this represents a different periodicity altogether or a change in the System IV period.

At Saturn, the almost axially aligned dipole means that longitudinal asymmetries in the magnetic field are small,

Published by Copernicus GmbH on behalf of the European Geosciences Union. 


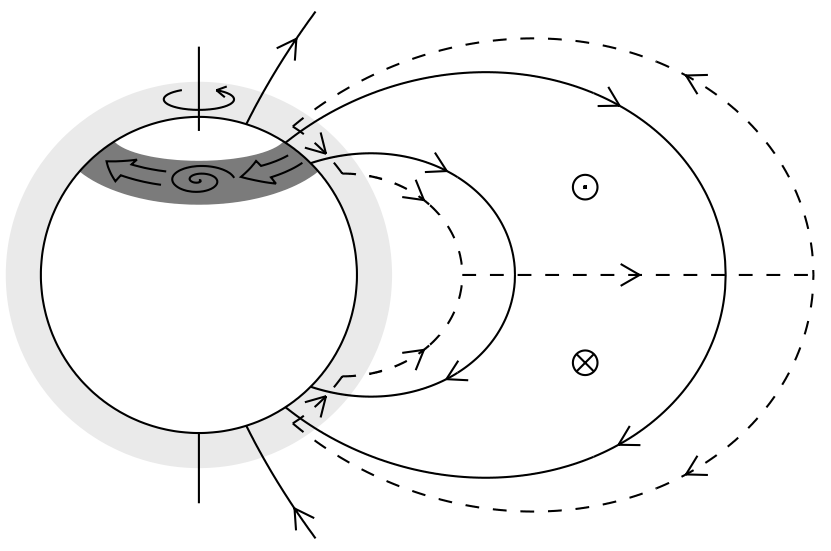

Fig. 1. Sketch of the Hill current system. Equatorward Pedersen currents in the ionosphere (light grey shaded region) close in the equatorial magnetosphere, acting to transfer angular momentum from the upper atmosphere to the magnetosphere. The currents (dashed lines) induce an azimuthal magnetic field which bends the planetary field lines (solid lines) out of the meridian planes. The region of the atmosphere coupled to this current system (dark grey shaded region) is expected to be dominated by subcorotational winds. However, an axial asymmetry in this region will lead to an axial asymmetry in the coupling currents and thus in the magnetic field perturbations.

and its rotation rate is difficult to determine (Giampieri and Dougherty, 2004). However, $\sim 10 \mathrm{~h}$ periodicities are observed in emissions of Saturn kilometric radiation (SKR; Desch and Kaiser, 1981). This radiation has subsequently been characterised in detail and the source regions located. Various mechanisms for the emission have been proposed (see review by Zarka and Kurth, 2005, and references therein). However, it is still not clear why it should exhibit a $\sim 10 \mathrm{~h}$ periodicity. The value of this periodicity has also changed at the $\sim 1 \%$ level since its discovery (Galopeau and Lecacheux, 2000). A possible explanation for this $\sim 1 \%$ level variability has recently been suggested by Cecconi and Zarka (2005) in terms of solar wind-magnetosphere coupling.

The periodicity at Saturn has also been detected in magnetometer data from the Voyager flybys (Espinosa et al., 2003a), and more recently by Cassini (Cowley et al., 2006; Giampieri et al., 2006). It is thus clear that it represents a periodic modulation of large regions of the magnetosphere, and is not confined to the SKR source region close to the planet. Espinosa et al. (2003b) have argued that some characteristics of the observed magnetic field signatures may be explained by an equatorial magnetic anomaly driving compressional Alfvèn waves into the magnetosphere. However, there is at present no evidence for such a feature, nor is it clear whether it is plausible for a magnetic anomaly generated deep in the planet to change its rotation velocity at the $1 \%$ level on a decadal timescale, or shorter.

Here we tentatively propose that the periodic signatures at both planets may originate in the dynamics of the neutral at- mosphere. This is possible because the neutral atmosphere is coupled to the magnetosphere in the conducting regions of the ionosphere. In Sect. 2 we outline why an atmospheric mechanism becomes possible when some common simplifying assumptions about magnetosphere-ionosphere coupling are discarded. In Sects. 3 and 4 we then discuss whether such a mechanism is plausible given our current knowledge of the Jovian and Kronian upper atmospheres, and then in Sect. 5 we argue that it has the potential to resolve some of the unexplained peculiarities mentioned above. Finally, in Sect. 6, we conclude.

\section{Proposed mechanism}

It has been argued in the context of Saturn (Espinosa et al., 2003b) that the neutral atmosphere cannot be responsible for communicating a single $\sim 10 \mathrm{~h}$ periodicity to the magnetosphere via the mechanism of Hill (1979). This follows from the observation, already noted, that different regions of the magnetosphere acquire different rotational velocities via this process. Furthermore, these rotational velocities are normally longer than $\sim 10 \mathrm{~h}$. Any physical asymmetry would be "frozen-in" to these sub-corotating flux tubes and could therefore not be responsible for the $\sim 10 \mathrm{~h}$ periodicity.

The coupled region of the upper atmosphere is also expected to subcorotate with respect to deeper layers of the atmosphere, since this velocity difference is supposedly necessary to allow the required upwards viscous transfer of angular momentum. Thus - it is argued - the $\sim 10 \mathrm{~h}$ periodicity cannot be imposed by the upper atmosphere, since the entire system subcorotates. However, this magnetospherecentred view of the thermosphere-ionosphere system implicitly makes two dangerous simplifying assumptions.

Firstly, it assumes that the only winds are the zonal (eastwest) winds that are induced by the magnetospheric drag (Huang and Hill, 1989; Pontius, 1995). The meridional (north-south) winds are assumed to be zero. This is difficult to justify. The fast rotation rates and large radii of the gas giants generate Coriolis forces $\sim 20$ times greater than those in the terrestrial system. Thus any zonal motion induced by ion drag must be subject to a substantial meridional Coriolis force. Excepting any extremely fortuitous circumstance in which some other meridional force - a pressure gradient, for example - balances this Coriolis force exactly, it seems inevitable that if there are zonal winds in the upper atmosphere, there will also be meridional winds.

The importance of this for the magnetosphere is that if there is a significant Hall conductivity, a meridional wind implies meridional Hall currents. From the perspective of the magnetosphere these currents are indistinguishable from the meridional Pedersen currents that mediate the transfer of angular momentum to the magnetosphere via the Hill mechanism. It is thus clear that, if there is sufficient Hall 
conductivity, meridional winds may drive current systems that perturb the magnetosphere.

Meridional winds may also play a role in the transport of angular momentum within the neutral atmosphere. If so, this may undermine the conventional understanding that angular momentum is supplied to the conducting layer by vertical viscous transport. Although interesting, this is not directly relevant to the argument of this paper, and will be discussed elsewhere (Smith, 2006).

The second problematic assumption is that the winds in the conducting layer of the upper atmosphere are axially symmetric. This is a useful simplifying assumption since it means that any zonal currents close exactly in the upper atmosphere. They thus have a negligible effect on the magnetosphere. However, if the flows in the upper atmosphere are not axially symmetric, then zonal currents may be divergent. They will thus drive field aligned currents that must close in, and perturb, the magnetosphere.

Furthermore, any asymmetry in the meridional or zonal winds will obviously manifest itself as an asymmetry in the currents flowing between the atmosphere and magnetosphere. This implies an axially asymmetric perturbation of the magnetosphere (Fig. 1). The details of this perturbation will depend on the distribution of the Pedersen and Hall conductivities in the upper atmosphere and the details of the asymmetry in the neutral winds.

It seems possible for such an asymmetric system of currents to impose a clear $\sim 10 \mathrm{~h}$ periodicity in the magnetosphere if two conditions are fulfilled. Firstly, the asymmetry in the neutral winds must be of sufficient magnitude, and coupled to sufficient conductivity, to drive perturbing currents into the magnetosphere that are at least of the same order of magnitude as the Hill mechanism currents. This is necessary because, otherwise, the periodic signature of the perturbing currents would be masked by the much greater Hill currents. Secondly, the asymmetry must rotate steadily with a unique $\sim 10 \mathrm{~h}$ period. We investigate these two conditions in turn.

\section{Conductivities and wind speeds}

The Pedersen and Hall conductivities $\left(\sigma_{P}\right.$ and $\left.\sigma_{H}\right)$ at any level of the upper atmosphere depend on the magnetic field $B$, the electronic charge $e$, the local ion and electron densities $\left(n_{i}\right.$ and $\left.n_{e}\right)$, the ion-neutral and electron-neutral collision frequencies $\left(v_{i n}\right.$ and $\left.v_{e n}\right)$ and the ion and electron gyrofrequencies $\left(\Omega_{i}\right.$ and $\left.\Omega_{e}\right)$. The standard expressions (e.g. Luhmann, $1995)$ are simplest when written in terms of the ratios between collision frequencies and gyrofrequencies $\left(r_{i}=v_{i n} / \Omega_{i}\right.$ and $r_{e}=v_{e n} / \Omega_{e}$ ):

$\sigma_{P}=\frac{n_{i} e}{B}\left[\frac{r_{e}}{1+r_{e}^{2}}+\frac{r_{i}}{1+r_{i}^{2}}\right]$
$\sigma_{H}=\frac{n_{i} e}{B}\left[\frac{1}{1+r_{e}^{2}}-\frac{1}{1+r_{i}^{2}}\right]$

These functions are clearly strongly dependent on $r_{i}$ and $r_{e}$. In general $r_{i}>r_{e}$, while both increase with decreasing altitude.

In Fig. 2a we plot the functions in square brackets in Eqs. (1) and (2), as a function of altitude, for Jupiter. The assumptions used to calculate these curves are the same as those used later in this section to estimate the Hall conductivity. The Pedersen conductivity function is strongly peaked at the altitudes $r_{i}=1$ and $r_{e}=1$. At altitudes above the level $r_{i}=1$ and below the level $r_{e}=1$ the Pedersen conductivity dominates over the Hall. In between the altitudes $r_{i}=1$ and $r_{e}=1$ is a region in which the Hall conductivity is dominant. This "Hall region" is shaded. Radio occultations (Lindal et al., 1985; Hinson et al., 1998) have shown that for both planets the peak ionosphere is approximately coincident with the upper Pedersen conducting region. At lower altitudes coincident with the Hall region sharp layers are observed in the electron density. Possible causes of these layers have been explored by Chen (1981) for Jupiter and Moses and Bass (2000) for Saturn. At lower altitudes still, coincident with the lower Pedersen region, there is at present no evidence for significant electron densities. These features of the ionosphere are shown schematically for Jupiter in Fig. $2 b$. Finally, Fig. $2 \mathrm{c}$ shows the relationship of the neutral atmosphere to the different conducting layers. The upper Pedersen conducting layer is seen to be coincident with the upper thermosphere, whereas the Hall region is coincident with the lower thermosphere and mesosphere. The situation at Saturn is similar.

We now move on to discuss the coupling between these conductivities and the neutral winds. To simplify the discussion that follows, we assume that the neutral wind velocities are independent of altitude throughout the Pedersen and Hall conducting layers, such that the behaviour is well parameterised by a single neutral wind speed interacting with heightintegrated conductivities $\Sigma_{P}=\int \sigma_{P} d z$ and $\Sigma_{H}=\int \sigma_{H} d z$. In practice this is likely to be a poor assumption, but it is sufficient for the order of magnitude estimates attempted here.

However, we do suppose that the neutral wind will vary with latitude and longitude. We split this variation into two components. Firstly, there must be a significant axisymmetric component of the neutral winds, $\boldsymbol{u}$, induced by subcorotational ion drag associated with the Hill mechanism currents. Secondly, we have our postulated non-axisymmetric perturbation $\delta \boldsymbol{u}$, the details of which we do not at present specify.

Assuming a vertical magnetic field $B$, height-integrated conductivities $\Sigma_{P}$ and $\Sigma_{H}$, and a zonal plasma drift velocity $v_{\phi}$, the meridional part of the axisymmetric component of the height-integrated current is given by

$$
J_{\theta}=\left(\Sigma_{P}\left(u_{\phi}-v_{\phi}\right)+\Sigma_{H} u_{\theta}\right) B=-\Sigma_{P}^{*} v_{\phi} B
$$


(a)

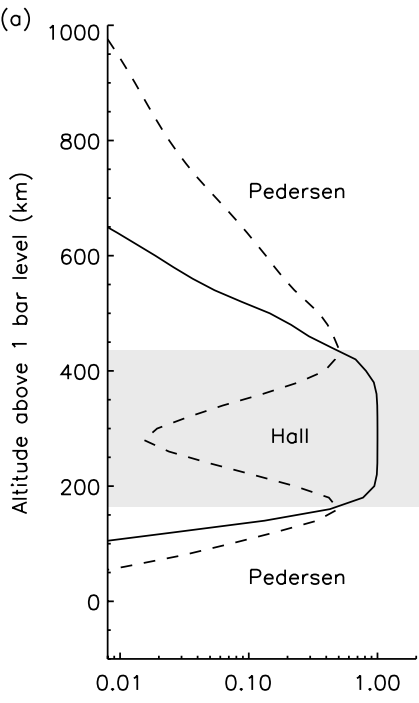

(b) (c)

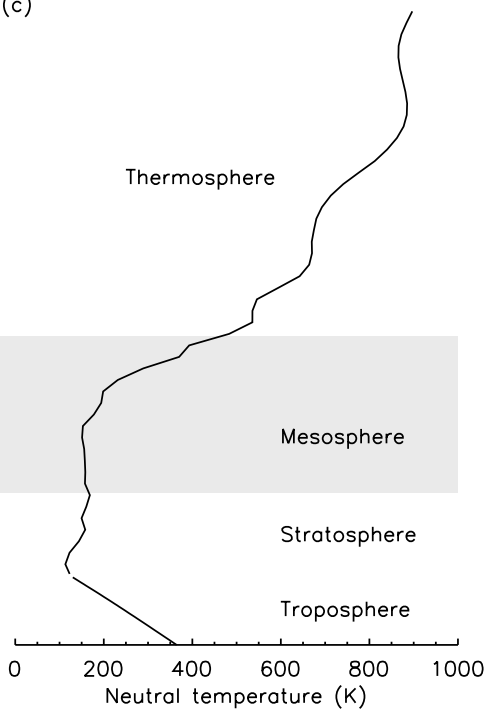

Fig. 2. Schematic of the conducting properties of Jupiter's atmosphere. (a) The functions in square brackets in Eqs. (1) and (2), representing the importance of Pedersen (dashed line) and Hall (solid line) conductivities. The shading shows the "Hall region" in which Hall conductivity is more important than Pedersen conductivity. At the upper boundary of the shaded region $r_{i}=1$ and at the lower boundary $r_{e}=1$. (b) Schematic of the ionospheric electron density structure. (c) Atmospheric temperature profile from the Galileo probe (Seiff et al., 1998).

where we have defined the effective pedersen conductivity $\Sigma_{P}^{*}=(1-K) \Sigma_{P}$ and $K$ is a normalised parameter representing the axisymmetric component of the neutral winds:

$K=\frac{u_{\phi}+\left(\Sigma_{H} / \Sigma_{P}\right) u_{\theta}}{v_{\phi}}$

The behaviour of the zonal and meridional winds involved in the Hill mechanism currents can thus be subsumed into the definition of $\Sigma_{P}^{*}$, provided $K$ is appropriately defined.

The magnitude of the total perturbation to the horizontal current $\delta \boldsymbol{J}$ driven by the non-axisymmetric component of the winds $\delta \boldsymbol{u}$ is approximately

$$
|\delta \boldsymbol{J}| \sim \Sigma|\delta \boldsymbol{u}| B
$$

where $\Sigma=\sqrt{\Sigma_{P}^{2}+\Sigma_{H}^{2}}$ is the total magnitude of the conductivity.

If either of these horizontal currents is divergent, it must close in and perturb the magnetosphere. We cannot assess the divergence of these currents without specifying details of the plasma and neutral velocities, which would make our argument less general. Therefore we make the simple assumption that both components drive currents into the magnetosphere roughly proportional to their magnitude. Thus, to meet our condition that the perturbing currents must be of the same order of magnitude as the Hill mechanism currents, we simply equate Eqs. (3) and (5):

$|\delta \boldsymbol{u}| \sim\left(\frac{\Sigma_{P}^{*}}{\Sigma}\right) v_{\phi}$
We may expect $v_{\phi}$ to be of the order of $1 \mathrm{~km} / \mathrm{s}$, depending on the latitude and the degree of corotation of the magnetosphere. Thus if the total conductivity is equal to the effective Pedersen conductivity we require similar perturbation velocities of the order of $1 \mathrm{~km} / \mathrm{s}$. However, unless $K \leq 0$, which seems unlikely in most circumstances, then we will always find $\Sigma>\Sigma_{P}^{*}$, and in general the perturbation velocity does not need to be as great as the plasma drift velocity for the perturbation currents to compete with the Hill mechanism currents. With this in mind we now estimate, based on our present knowledge of $\Sigma_{P}^{*}$ and $\Sigma$, the required values of $|\delta \boldsymbol{u}|$.

First, suppose that the Hall conductivity is negligible, so that $\Sigma \simeq \Sigma_{P}$. Then our condition reduces to

$|\delta \boldsymbol{u}| \sim(1-K) v_{\phi}$

and our assessment depends not on the absolute value of the conductivity, but on the response of the axisymmetric component of the neutral winds to the plasma velocity, represented by $K$. For Jupiter $K$ has been estimated, using the JIM model, to be $\sim 0.5$ (Millward et al., 2005), implying $|\delta \boldsymbol{u}| \sim 500 \mathrm{~m} / \mathrm{s}$. For Saturn, results from a version of the STIM model of the Kronian thermosphere (Müller-Wodarg et al., 2006), run to near steady-state, suggest that at Saturn $K \sim 0.5$ is also a reasonable estimate, but that in some circumstances it may be $\sim 0.95$ or greater at high latitudes (Smith, 2006). For Saturn we thus require, as a preliminary estimate, $|\delta \boldsymbol{u}|$ in the range $50-500 \mathrm{~m} / \mathrm{s}$.

Next we consider the possible contribution of $\Sigma_{H}$. Due to the presumed importance of zonal winds and meridional Pedersen currents, calculations of the Hall conductivity at 
either Jupiter or Saturn are rare in the literature. We may derive an approximate expression for $\Sigma_{H}$ by height-integrating Eq. (2) across the Hall region, noting that in this region the expression in square brackets is approximately unity. Thus, if the column density of ions (or, equivalently, electrons) in the Hall region is $N$, then we find:

$\Sigma_{H} \simeq \frac{N e}{B} \simeq 1.5 \mathrm{mho} \times\left(\frac{N}{1 \times 10^{14} \mathrm{~m}^{-2}}\right)\left(\frac{1 \times 10^{-5} \mathrm{~T}}{B}\right)$

Using this formula we can estimate $\Sigma_{H}$ for Jupiter and Saturn based on electron density profiles from radio occultations.

First we must determine the location of the Hall region at both planets. To do this, we must make some assumptions about ion composition to calculate the ratio $r_{i}$. At both Jupiter and Saturn the Hall region lies largely below the homopause in a region believed to be dominated by hydrocarbon or metallic ions (Kim and Fox, 1994; Moses and Bass, 2000). We thus assume that the ionosphere is dominated by ions with a molecular mass of $24 \mathrm{amu}$, equal to the atomic mass of $\mathrm{Mg}^{+}$(the metallic ion modelled by Moses and Bass, 2000) and representative of the molecular mass of simple hydrocarbons. To calculate $v_{i n}$ and $v_{e n}$ we use the expressions from Banks and Kockarts (1973) and Geiss and Bürgi (1986), respectively. Note that assuming a lower ion mass would tend to push the upper boundary of the Hall region to lower altitudes. This would have the effect of slightly reducing the Hall conductivities estimated below.

For Jupiter we assume $B \simeq 80 \times 10^{-5} \mathrm{~T}$ and use the neutral densities from Seiff et al. (1998), placing the Hall region in the range $160-420 \mathrm{~km}(\sim 500-0.1 \mu \mathrm{bar})$. For the electron densities, we use the reanalysis of the Voyager 2 radio occultations by Hinson et al. (1998). The exit occultation shows electron densities of $\sim 3 \times 10^{10} \mathrm{~m}^{-3}$, with some substructure, in the range $200-420 \mathrm{~km}$, corresponding to a column density in the Hall region of $N \sim 7 \times 10^{15} \mathrm{~m}^{-2}$. The entry occultation shows an electron density increasing roughly linearly with altitude in the range $300-420 \mathrm{~km}$, with an average value of $\sim 5 \times 10^{10} \mathrm{~m}^{-3}$, corresponding to a column density of $N \sim 6 \times 10^{15} \mathrm{~m}^{-2}$. Using the formula above, these two values for $N$ imply a Hall conductivity of approximately 11.5 mho. The results of Hill (1980) imply that $\Sigma_{P}^{*} \sim 0.03$ mho at Jupiter if the mass outflow rate from Io is $1000 \mathrm{~kg} / \mathrm{s}$. Using these numbers in Eq. (6) implies $|\delta \boldsymbol{u}| \sim 20-30 \mathrm{~m} / \mathrm{s}$.

For Saturn we assume $B \simeq 6 \times 10^{-5} \mathrm{~T}$ and use the neutral densities from Moses et al. (2000), placing the Hall region in the range $470-1000 \mathrm{~km}(\sim 40-0.006 \mu$ bar $)$. Evidence for ionisation in the Hall region is again provided by the Voyager radio occultation experiments, which show layered structures in the electron density at and below $1000 \mathrm{~km}$ altitude (Lindal et al., 1985). We note that similar profiles from the Cassini mission have recently been published (Nagy et al., 2006), but in their published form they do not contain sufficient detail at low altitude to be of use here. By the above formula, a layer with a vertical width of $10 \mathrm{~km}$ and an average electron density of $1 \times 10^{10} \mathrm{~m}^{-3}$ corresponds to $\sim 0.25$ mho of Hall conductivity. There are a number of layers visible in the data, some considerably wider than $10 \mathrm{~km}$ and some with a density greater than $5 \times 10^{11} \mathrm{~m}^{-3}$. Taking these structures as a whole, we might expect the total Hall conductivity to be at least $1 \mathrm{mho}$ and probably no greater than $5 \mathrm{mho}$. These values are of the same order of magnitude as the limit $\Sigma_{P}^{*}<2$ mho derived by Bunce et al. (2003). Thus, unless $\Sigma_{P}^{*}$ is considerably less than this limit, we require $|\delta \boldsymbol{u}| \sim 0.5-1 \mathrm{~km} / \mathrm{s}$.

The estimates above show that, given our current knowledge, the required velocities are much smaller at Jupiter than at Saturn. The best case scenario at Jupiter requires winds of only $\sim 20 \mathrm{~m} / \mathrm{s}$ coupled to the Hall conductivity, and these estimates are based on reasonably reliable, published data. At Saturn the best case scenario requires winds of $\sim 50 \mathrm{~m} / \mathrm{s}$ in the Pedersen conducting layer and a large value of $K$ whose only justification is unpublished numerical modelling results. Nevertheless, these best case estimates compare well with observed wind velocities at both planets. For example, the latitudinal variability of the zonal winds in Jupiter's lower atmosphere is of the order of $100 \mathrm{~m} / \mathrm{s}$ at the equator and $50 \mathrm{~m} / \mathrm{s}$ at mid latitudes; circulation velocities in the great red spot (GRS) are of the order of $100 \mathrm{~m} / \mathrm{s}$ (Ingersoll et al., 2004). If similar asymmetric structures are present in the middle atmosphere, these velocities are more than adequate to provide the necessary magnetospheric perturbations. At Saturn, Hubbard et al. (1997) showed that equatorial winds of $\sim 450 \mathrm{~m} / \mathrm{s}$ persisted into the mesosphere region, and we would expect these winds to exhibit some asymmetries analagous to those observed in the lower atmosphere.

It is worth noting that our estimates of $\Sigma$ are almost exclusively based on low- and mid-latitude radio occultations. In the auroral regions of Jupiter and Saturn high-energy precipitating particles generate significant additional ionisation. At both planets most of the magnetosphere is coupled magnetically either directly to these regions or to regions that lie within a few degrees of latitude. The determinations of $\Sigma_{P}^{*}$ mentioned above relate largely to these regions. Thus, if we expect the coupling currents to be most important in these high latitude regions then we are likely to be overestimating the ratio $\Sigma_{P}^{*} / \Sigma$. We must therefore consider the possibility that precipitation in the auroral zones may reduce our required value of $|\delta \boldsymbol{u}|$ further.

Millward et al. (2002) used the JIM model of the Jovian upper atmosphere to calculate enhancements in the Pedersen and Hall conductivities due to electron precipitation. They found that the Pedersen was enhanced much more than the Hall - however, this is largely due to the location of the lower boundary of the model, which lies close to the top of the Hall region. They also found that for electron energies greater than $60 \mathrm{keV}$ the particles were energetic enough to penetrate below the lower boundary, into the Hall region. We expect similar behaviour at Saturn. We might thus speculate that in the auroral regions the postulated hydrocarbon and metallic 


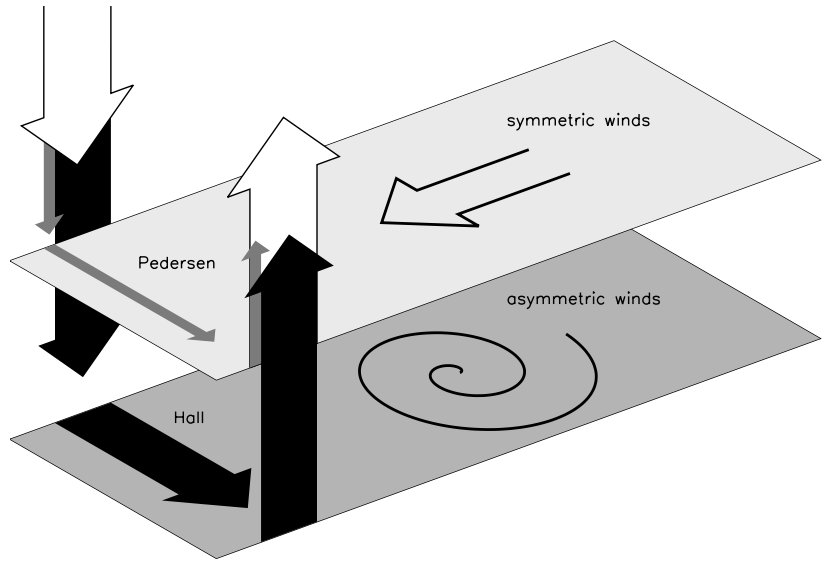

Fig. 3. Schematic of ionospheric current systems for an asymmetry in the Hall region. Axisymmetric neutral winds in the Pedersen conducting layer - expected to be primarily subcorotational - are associated with the Hill current system that transfers angular momentum to the magnetosphere (grey arrrows). However, if the Hall conductivity is significant, then asymmetric winds in this layer which need not be subcorotational - may also drive currents into the magnetosphere (black arrows). If these currents are greater than the Hill currents, they may dominate the total current (white arrows) and thus dominate the perturbation to the magnetospheric fields.

ion layers at low altitudes will be considerably enhanced, by precipitation, over those seen in the low- and mid-latitude occultations. It should be emphasised that although these layers are observed in radio occultations and predicted by models, they have yet to be detected spectroscopically.

The time constant for the recombination of metallic ions such as $\mathrm{Mg}^{+}$is also significantly longer than that for the $\mathrm{H}^{+}$and $\mathrm{H}_{3}^{+}$ions that dominate the peak ionosphere (Moses and Bass, 2000). This suggests that if their density were enhanced in the auroral regions there would be adequate time for them to be transported either by diffusion or by meridional winds to nearby regions of the upper atmosphere that are not directly ionised by the aurora. Transport by winds seems particularly likely, given that in the Hall region the motion of the ions is almost fully coupled to the neutral winds. This would enhance the Hall conductivity away from the immediate zone of precipitation. In contrast, the $\mathrm{H}^{+}$and $\mathrm{H}_{3}^{+}$ions that are associated with the Pedersen conductivity are not coupled sufficiently to the neutral winds, nor are their recombinative time constants long enough, for their densities to be enhanced in regions not subject to continuous ionisation. Thus we might expect a region close to the aurora that exhibits a Hall conductivity significantly enhanced over the effective Pedersen conductivity. Equation (6) shows that this would allow relatively small perturbations in the Hall region to dominate the ionospheric current systems at these latitudes.

Finally, it is worth speculating on the behaviour of an asymmetry in the equatorial regions of either planet. In these regions the connected magnetosphere is forced into corotation easily by the Hill mechanism, and there is thus no requirement for the Pedersen conducting layer to subcorotate. Furthermore, the ionospheric structure is likely to be complex, due to the near horizontal equatorial magnetic field. Winds in this region may thus drive significant perturbing current systems in the very inner magnetosphere. These perturbations might then propagate through the magnetosphere by the same mechanism described by Espinosa et al. (2003b) in the context of an equatorial magnetic field anomaly.

\section{Rotation period}

Our second condition is that the asymmetry must be persistent, and must rotate with a unique $\sim 10 \mathrm{~h}$ period. Such an asymmetry might be a very localised, self-sustaining wind system (a "storm") or a larger scale, global asymmetry (a "planetary wave"). Our best model of such a persistent feature, corresponding in particular to the former case, is the GRS, which has persisted in the Jovian troposphere for greater than $\sim 150$ years, with an approximately corotational velocity (Ingersoll et al., 2004). We will not focus further here on the possible nature of such systems: rather we will try to convince the reader that, if such an asymmetry exists, it may communicate its rotation period to the magnetosphere.

If an asymmetric wind system lies directly in the Pedersen conducting layer, then it probably does not fulfil our criteria. This is because the layer is expected to subcorotate significantly to allow the upwards viscous transfer of angular momentum that balances ion drag (Huang and Hill, 1989; Pontius, 1995). Thus, unless the corotation lag is tiny, the period of any asymmetry in this layer would be considerably greater than $\sim 10 \mathrm{~h}$.

However, a corotating asymmetry lying below the subcorotating Pedersen conducting layer may impose a signature on that layer by generating a "wake" whose form is fixed with respect to corotation. Such a "wake" is analagous to the formation of atmospheric waves in the lee of a mountain: the form of the waves may be fixed with respect to the mountain, even though the flow containing the waves is not. Thus it is possible, in principle, for a corotating asymmetry to be coupled to the Pedersen conductivity.

More plausible, though, is a wind system coupled to the Hall conductivity, sketched in Fig. 3. The Hall conducting region is not required to subcorotate, and thus our corotating feature may exist directly in the Hall region and couple directly to the Hall conductivity. If the Hall conductivity is enhanced close to the aurora, as discussed above, then even a small asymmetry in the winds in this region might suffice. This region of the neutral atmosphere corresponds to the mesosphere and lower thermosphere at both Jupiter and Saturn. The author is not aware of any studies of middle atmosphere dynamics at Jupiter or Saturn that might rule out the existence of a corotating asymmetry in this region; indeed, 
there is very little data to constrain such studies. However, it may be interesting to consider whether the complex energy balance of the mesosphere - consisting presumably of a mixture of solar heating, infrared radiative cooling, breaking gravity waves, advection, thermal conduction and, at high latitudes, possible heating from precipitating energetic particles - may give rise to persistent localised wind systems such as those proposed here.

Alternatively, the feature may exist at lower altitudes still - in the stratosphere or troposphere - and impose its rotation period on the conducting region of the upper atmosphere through vertical coupling processes. Depending on the location and nature of the feature, it may drive other layers of the atmosphere either by simple viscous coupling, by vertical convective coupling, or by the generation, propagation and dissipation of gravity waves.

Finally, it is worth emphasising our contention that an asymmetry in the upper atmospheric winds may exist independently of any asymmetry in the planet's internal magnetic field. The processes that drive the upper atmosphere - coupling to the lower atmosphere and magnetosphere; solar insolation - are sufficiently complicated and variable to ensure that the upper atmosphere is not a perfectly axisymmetric system. The important question is whether there exist welldefined, persistent asymmetries of the appropriate magnitude and morphology to produce the observed effects.

\section{Comparison with observations}

Various of the unexplained features of the periodicities can be understood rather easily in terms of our mechanism. We know from studies of the GRS that atmospheric vortices can be persistent and also exhibit variability (Ingersoll et al., 2004). Such variability naturally explains the $\sim 1 \%$ level change in the SKR period and the possible $\sim 1.5 \%$ change in the System IV period suggested by the results of Steffl et al. (2006).

In the case of Jupiter, atmospheric variability could also explain the phase jumps exhibited by the System IV period. There may, as suggested by Woodward et al. (1997), be two corotating features separated in longitude whose relative magnitude changes periodically. If the features are atmospheric this seems very plausible. Alternatively, it may be that an asymmetry is a meta-stable state of the upper atmosphere at some latitude. One could then envisage the asymmetry persisting stably for several years, suddenly breaking up, and then rapidly reforming in a different longitude sector. The difference between the type of periodic signatures due to System III and System IV may also be explained by our mechanism if the System III is imposed by the magnetic field asymmetry and System IV by an atmospheric asymmetry. Without knowledge of the details of the interactions it is likely that these two mechanisms would produce different signatures.
Finally, our mechanism can explain the pulsed nature of SKR. A corotating system of the sort described would enter the SKR source region every $\sim 10 \mathrm{~h}$, altering the coupling currents in this region and perhaps triggering radio emission. One problem with this interpretation is that if the atmospheric feature is at a high latitude it must somehow trigger SKR emission in both hemispheres. This would either imply a small time delay between the emission from opposite hemispheres, or require similar systems in each hemisphere that are correlated in longitude. The latter seems unlikely.

\section{Conclusions}

We have argued that the neutral atmosphere may be responsible for some of the unexplained periodic signatures observed at Jupiter and Saturn. Our argument is very general because our understanding of the planets' upper atmospheres is at present rather threadbare. With the limited data available, we have shown that currents driven by a non-axisymmetric component of the upper atmospheric winds may, under some circumstances, be comparable to the axisymmetric Hill mechanism currents. However, the required wind velocities are critically dependent on the true Pedersen and Hall conductivities, which are very uncertain. In particular, there is very little data to constrain the true conductivities in the auroral zones, where the coupling between the atmosphere and magnetosphere is likely to be most pronounced. Nevertheless, our mechanism can explain variability in the SKR and System IV periods, and has the potential, with further work, to explain some of the other unexplained periodic phenomena.

This brief study therefore presents a number of interesting questions. Most importantly, a sound understanding of the ionosphere at equatorial and auroral latitudes is long overdue. Cassini will hopefully provide a comprehensive set of radio occultations, at numerous latitudes, that may help to resolve this problem at Saturn. A more complex problem is the dynamical behaviour of the middle atmosphere. Given the highly visible complexity of tropospheric dynamics at both planets, it seems improbable that the middle atmosphere will be entirely quiescent. There is, however, minimal data to constrain the behaviour of this region. Until this situation improves, any progress is likely to be based largely on intelligent speculation.

Finally, given structures in the ionised and neutral atmospheres that generate current systems, it is necessary to understand the extent to which these currents are divergent, and how they then close in and perturb the magnetosphere. We must also consider the possibility that, treating the entire system from the stratosphere to the magnetopause as a single entity, there may be positive feedback effects within the system that reinforce or encourage asymmetries. 
Acknowledgements. This work was supported by a CASE studentship from the UK Particle Physics and Astronomy Research council and Sun Microsystems Ltd. I am also grateful to A. Aylward, S. Miller and others at UCL for numerous helpful comments and suggestions.

Topical Editor I. A. Daglis thanks two referees for their help in evaluating this paper.

\section{References}

Banks, P. M. and Kockarts, G.: Aeronomy, Academic Press, 1973.

Brown, M. E.: Periodicities in the Io plasma torus, J. Geophys Res., 100, $21683-21696,1995$.

Bunce, E. J., Cowley, S. W. H., and Wild, J. A.: Azimuthal magnetic fields in Saturn's magnetosphere: effects associated with plasma subcorotation and the magnetopause-tail current system, Ann. Geophys., 21, 1709-1722, 2003.

Cecconi, B. and Zarka, P.: Model of a variable radio period for Saturn, J. Geophys Res., 110, 12 203, doi:10.1029/2005JA011085, 2005.

Chen, R. H.: Studies of Jupiter's lower ionospheric layers, J. Geophys Res., 86, 7792-7794, 1981.

Cowley, S. W. H., Wright, D. M., Bunce, E. J., Carter, A. C., Dougherty, M. K., Giampieri, G., Nichols, J. D., and Robinson, T. R.: Cassini observations of planetary-period magnetic field oscillations in Saturn's magnetosphere: Doppler shifts and phase motion, Geophys. Res. Lett., 33, doi:10.1029/2005GL025522, 2006.

Desch, M. D. and Kaiser, M. L.: Voyager measurement of the rotation period of Saturn's magnetic field, Geophys. Res. Lett., 8, 253-256, 1981.

Espinosa, S. A., Southwood, D. J., and Dougherty, M. K.: Reanalysis of Saturn's magnetospheric field data view of spin-periodic perturbations, J. Geophys Res., 108, doi:10.1029/2001JA005083, 2003a.

Espinosa, S. A., Southwood, D. J., and Dougherty, M. K.: How can Saturn impose its rotation period in a noncorotating magnetosphere?, J. Geophys Res., 108, doi:10.1029/2001JA005084, 2003 b.

Galopeau, P. H. M. and Lecacheux, A.: Variations of Saturn's radio rotation period measured at kilometer wavelengths, J. Geophys Res., 105, 13 089-13 102, 2000.

Geiss, J. and Bürgi, A.: Diffusion and thermal diffusion in partially ionized gases in the atmospheres of the sun and planets, Astronomy \& Astrophysics, 159, 1-2, 1986.

Giampieri, G. and Dougherty, M. K.: Rotation rate of Saturn's interior from magnetic field observations, Geophys. Res. Lett., 31, 16 701, doi:10.1029/2004GL020194, 2004.

Giampieri, G., Dougherty, M. K., Smith, E. J., and Russell, C. T.: A regular period for Saturn's magnetic field that may track its internal rotation, Nature, 441, doi:10.1038/nature04750, 2006.

Hill, T. W.: Inertial limit on corotation, J. Geophys Res., 84, 65546558, 1979.

Hill, T. W.: Corotation lag in Jupiter's magnetosphere - Comparison of observation and theory, Science, 207, 301-302, 1980.

Hinson, D. P., Twicken, J. D., and Karayel, E. T.: Jupiter's ionosphere: New results from Voyager 2 radio occultation measurements, J. Geophys Res., 103, 9505-9520, 1998.
Huang, T. S. and Hill, T. W.: Corotation lag of the Jovian atmosphere, ionosphere, and magnetosphere, J. Geophys Res., 94, 3761-3765, 1989.

Hubbard, W. B., Porco, C. C., Hunten, D. M., Rieke, G. H., Rieke, M. J., McCarthy, D. W., Haemmerle, V., Haller, J., McLeod, B., Lebofsky, L. A., Marcialis, R., Holberg, J. B., Landau, R., Carrasco, L., Elias, J., Buie, M. W., Dunham, E. W., Persson, S. E., Boroson, T., West, S., French, R. G., Harrington, J., Elliot, J. L., Forrest, W. J., Pipher, J. L., Stover, R. J., Brahic, A., and Grenier, I.: Structure of Saturn's Mesosphere from the 28 SGR Occultations, Icarus, 130, 404-425, 1997.

Ingersoll, A. P., Dowling, T. E., Gierasch, P. J., Orton, G. S., Read, P. L., Sánchez-Lavega, A., Showman, A. P., Simon-Miller, A. A., and Vasavada, A. R.: Dynamics of Jupiter's atmosphere, pp. 105-128, Jupiter. The Planet, Satellites and Magnetosphere, 2004.

Kim, Y. H. and Fox, J. L.: The chemistry of hydrocarbon ions in the Jovian ionosphere, Icarus, 112, 310-325, 1994.

Lindal, G. F., Sweetnam, D. N., and Eshleman, V. R.: The atmosphere of Saturn - an analysis of the Voyager radio occultation measurements, Astronomical Journal, 90, 1136-1146, 1985.

Luhmann, J. G.: Ionospheres, pp. 183-202, in: Introduction to Space Physics, edited by: Kivelson, M. G. and Russell, C. T., Cambridge University Press, 1995.

McNutt, R. L., Belcher, J. W., Sullivan, J. D., Bagenal, F., and Bridge, H. S.: Departure from rigid co-rotation of plasma in Jupiter's dayside magnetosphere, Nature, 280, 803-804, 1979.

Millward, G., Miller, S., Stallard, T., Aylward, A. D., and Achilleos, N.: On the Dynamics of the Jovian Ionosphere and Thermosphere III: The Modelling of Auroral Conductivity, Icarus, 160, 95-107, 2002.

Millward, G., Miller, S., Stallard, T., Achilleos, N., and Aylward, A. D.: On the dynamics of the jovian ionosphere and thermosphere IV: Ion-neutral Coupling, Icarus, 173, 200-211, 2005.

Moses, J. I. and Bass, S. F.: The effects of external material on the chemistry and structure of Saturn's ionosphere, J. Geophys Res., 105, 7013-7052, 2000.

Moses, J. I., Bézard, B., Lellouch, E., Gladstone, G. R., Feuchtgruber, H., and Allen, M.: Photochemistry of Saturn's Atmosphere. I. Hydrocarbon Chemistry and Comparisons with ISO Observations, Icarus, 143, 244-298, 2000.

Müller-Wodarg, I. C. F., Mendillo, M., Yelle, R. V., and Aylward, A. D.: A global circulation model of Saturn's thermosphere, Icarus, 180, 147-160, 2006.

Nagy, A. F., Kliore, A. J., Marouf, E., French, R., Flasar, M., Rappaport, N. J., Anabtawi, A., Asmar, S. W., Johnston, D., Barbinis, E., Goltz, G., and Fleischman, D.: First results from the ionospheric radio occultations of Saturn by the Cassini spacecraft, J. Geophys Res., 111, doi:10.1029/2005JA011519, 2006.

Pontius, D. H.: Implications of variable mass loading in the Io torus: The Jovian flywheel, J. Geophys Res., 100, 19531-19540, 1995.

Richardson, J. D.: Thermal ions at Saturn - Plasma parameters and implications, J. Geophys Res., 91, 1381-1389, 1986.

Sandel, B. R. and Dessler, A. J.: Dual periodicity of the Jovian magnetosphere, J. Geophys Res., 93, 5487-5504, 1988.

Seiff, A., Kirk, D. B., Knight, T. C. D., Young, R. E., Mihalov, J. D., Young, L. A., Milos, F. S., Schubert, G., Blanchard, R. C., and Atkinson, D.: Thermal structure of Jupiter's atmosphere near the edge of a $5-\mu \mathrm{m}$ hot spot in the north equatorial belt, J. Geophys 
Res., 103, 22 857-22 890, 1998.

Smith, C. G. A.: Modelling the Thermospheres of the Giant Planets, PhD thesis, UCL, 2006.

Steffl, A. J., Delamere, P. A., and Bagenal, F.: Cassini UVIS observations of the Io plasma torus, Icarus, 180, 124-140, 2006.
Woodward, R. C. J., Scherb, F., and Roesler, F. L.: Variations in Optical $\mathrm{S}^{+}$Emission from the Io Plasma Torus: Evidence for Quasi Periodicity, Astrophysical Journal, 479, 984-991, 1997.

Zarka, P. and Kurth, W. S.: Radio Wave Emission from the Outer Planets Before Cassini, Space Sci. Rev., 116, 371-397, 2005. 